\title{
A POLYNOMIAL CLASS OF MARKUS-YAMABE COUNTEREXAMPLES
}

\author{
Anna Cima, Armengol Gasull and Francesc Mañosas
}

\begin{abstract}
In the paper [CEGHM] a polynomial counterexample to the Markus-Yamabe Conjecture and to the discrete Markus-Yamabe Question in dimension $n \geq 3$ are given. In the present paper we explain a way for obtaining a family of polynomial counterexamples containing the above ones. Finally we study the global dynamics of the examples given in [CEGHM].
\end{abstract}

\section{Introduction}

Let $F: \mathbb{R}^{n} \longrightarrow \mathbb{R}^{n}$ be a $\mathcal{C}^{1}$ map and consider the differential system

$$
\dot{x}=F(x) \text {. }
$$

Assume that $p$ is a critical point of $(1)$, i.e., $F(p)=0$. We say that $p$ is a global attractor of the continuous dynamical system $(1)$ if $\phi(t, x)$ is defined for all $t>0$ and tends to $p$ as $t$ tends to infinity for each $x \in \mathbb{R}^{n}$, where $\phi(t, x)$ is the solution of (1) with initial condition $\phi(0, x)=x$.

The next conjecture was explicitly stated by Markus and Yamabe (see [MY]) in 1960.

MYC(n) (Markus-Yamabe Conjecture). Let $F$ be a $\mathcal{C}^{1}$ vector field defined on $\mathbb{R}^{n}$ such that for any $x \in \mathbb{R}^{n}$, the jacobian of $F$ at $x$ has all its eigenvalues with negative real part. If $F(p)=0$, then $p$ is a global attractor of $\dot{x}=F(x)$.

This conjecture was proved for planar polynomial maps in 1988 (see [MO]) and for planar $\mathcal{C}^{1}$ maps in 1993 (see $[\mathbf{F}]$ and $[\mathbf{G u}]$ ) and in 1994 (see $[\mathbf{G 1}])$. In $[\mathbf{B}]$ and $[\mathbf{B L}]$ there are examples of smooth vector fields defined in $\mathbb{R}^{n}, n \geq 4$ satisfying the hypothesis of the Conjecture and having a periodic orbit. On the other hand in [CEGHM], the authors 
give an example of a polynomial vector field in $\mathbb{R}^{n}$ for $n \geq 3$ satisfying the hypothesis of the MYC such that it has orbits which scape at infinity. A non polynomial counterexample for $n \geq \mathbb{R}^{3}$ is presented in [G2].

The goal of this paper is to give a more general family of polynomial counterexamples and explain how they are obtained. The construction of the counterexamples is based on two points: The first one concerns on the characterizacion of vector fields which have solutions of exponential type, that lead us with the notion of linear quasi-homogeneous vector fields (see Section 2). The second one deals with the construction of nilpotent maps (see Section 3).

Using the same tools we can also give an answer to the Discrete Markus-Yamabe Question, which can be established as follows.

Let $F: \mathbb{R}^{n} \longrightarrow \mathbb{R}^{n}$ be a $\mathcal{C}^{1}$ map and consider the sequence:

$$
x^{(m+1)}=F\left(x^{(m)}\right), \quad x^{(0)} \in \mathbb{R}^{n} .
$$

Now consider the dynamics of the iterations of $F$. Let $p$ be a fixed point of $F$, i.e., $F(p)=p$. We say that $p$ is a global attractor of the discrete dynamical system (2) if the sequence $x^{(m)}$ tends to $p$ when $m$ tends to infinity for any $x^{(0)} \in \mathbb{R}^{n}$. The question is the following:

DMYQ(n) (Discrete Markus-Yamabe Question). Let $F$ be a $\mathcal{C}^{1}$ map from $\mathbb{R}^{n}$ into itself such that $F(0)=0$ and for any $x \in \mathbb{R}^{n}$, $J F(x)$ has all its eigenvalues with modulus less than one. Is it true that 0 is a global attractor for the discrete dynamical system generated by $F$ ?

The above problem is introduced in $[\mathbf{S}]$ and $[\mathbf{C G M}]$. In this last paper the authors prove that the answer is negative even in the planar case and that the conclusion is affirmative if we take into account polynomial maps defined in $\mathbb{R}^{2}$. The planar example which gives a negative answer to this question has a periodic orbit of period 4 and is given by a rational function. In $[\mathbf{E H}]$ the authors give a negative answer to this question for polynomial maps in dimension greather than three. A polynomial counterexample for dimension greather than two is presented in [CEGHM].

In this paper we also give a family of polynomial maps in $\mathbb{R}^{n}$ for $n \geq 3$ satisfying the hypothesis of DMYQ(n) and such that there are some unbounded orbits.

The paper is organized as follows. In the next section we give some results on quasi-homogeneous vector fields of degree one. In Section 3 we 
construct the mentioned families of counterexamples. The last section is devoted to study the global dynamics of the simplest counterexamples inside the family (the ones given in [CEGHM]).

This paper is partially supported by the DGICYT grant number PB93-0860.

\section{On quasi-homogeneous vector fields of degree one}

This section is a development of some results of [A, Chap. 1]. We say that $f: \mathbb{R}^{n} \rightarrow \mathbb{R}$ is a quasi-homogeneous function with weigths $\alpha_{1}, \alpha_{2}, \ldots, \alpha_{n}$ and quasi-degree $d$ if

$$
f\left(\lambda^{\alpha_{1}} x_{1}, \lambda^{\alpha_{2}} x_{2}, \ldots, \lambda^{\alpha_{n}} x_{n}\right)=\lambda^{d} f\left(x_{1}, x_{2}, \ldots, x_{n}\right),
$$

for all $\lambda>0$ and for all $x \in \mathbb{R}^{n}$. We stress that the weights can be taken as non zero real numbers.

Notice that if $f$ is a quasi-homogeneous function with weights $\alpha_{1}, \alpha_{2}, \ldots, \alpha_{n}$ and quasi-degree $d$ then, for all constant $c \in \mathbb{R}, c \neq 0, f$ is also a quasi-homogeneous function with weights $c \alpha_{1}, c \alpha_{2}, \ldots, c \alpha_{n}$ and quasi-degree $c d$.

On the other hand let $f$ be a quasi-homogeneous analytic function with weights $\alpha_{1}, \alpha_{2}, \ldots, \alpha_{n}$ and write $f$ as a sum of monomials:

$$
f(x)=\sum_{r_{1}, r_{2}, \ldots, r_{n}} A_{r_{1} r_{2} \ldots r_{n}} x_{1}^{r_{1}} x_{2}^{r_{2}} \cdots x_{n}^{r_{n}} .
$$

Since for all $\lambda>0(3)$ is satisfied, we have that

$$
\begin{aligned}
\sum_{r_{1}, r_{2}, \ldots, r_{n}} A_{r_{1} r_{2} \ldots r_{n}} x_{1}^{r_{1}} x_{2}^{r_{2}} \cdots x_{n}^{r_{n}} \lambda^{\left(r_{1} \alpha_{1}+r_{2} \alpha_{2}+\cdots+r_{n} \alpha_{n}\right)} & \\
& =\lambda^{d} \sum_{r_{1}, r_{2}, \ldots, r_{n}} A_{r_{1} r_{2} \ldots r_{n}} x_{1}^{r_{1}} x_{2}^{r_{2}} \cdots x_{n}^{r_{n}} .
\end{aligned}
$$

Hence, the only monomials which appear in the decomposition of $f$ are of the form $x_{1}^{r_{1}} x_{2}^{r_{2}} \cdots x_{n}^{r_{n}}$ with $r_{1} \alpha_{1}+r_{2} \alpha_{2}+\cdots+r_{n} \alpha_{n}=d$.

We say that $F=\left(F_{1}, F_{2}, \ldots, F_{n}\right): \mathbb{R}^{n} \rightarrow \mathbb{R}^{n}$ is a quasi-homogeneous vector field with weights $\alpha_{1}, \alpha_{2}, \ldots, \alpha_{n}$ and quasi-degree $d$ if each $F_{i}$ is a quasi-homogeneous function with weights $\alpha_{1}, \alpha_{2}, \ldots, \alpha_{n}$ and quasidegree $\alpha_{i}+d-1$, see [BDST, Chap. 7].

We say that $F$ is a linear quasi-homogeneous vector field if it is a quasi-homogeneous vector field of degree one.

From now on we deal with linear quasi-homogeneous vector fields. 
Proposition 2.1. Let $F$ be a linear quasi-homogeneous vector field with weights $\alpha_{1}, \alpha_{2}, \ldots, \alpha_{n}$ and consider the differential system $\dot{x}=$ $F(x)$. Then $F$ is invariant by the change $\bar{x}_{i}=\lambda^{\alpha_{i}} x_{i}$.

Proof: We have to show that if $x(t)=\left(x_{1}(t), x_{2}(t), \ldots, x_{n}(t)\right)$ is a solution of $\dot{x}=F(x)$ then $\bar{x}(t)=\left(\lambda^{\alpha_{1}} x_{1}(t), \lambda^{\alpha_{2}} x_{2}(t), \ldots, \lambda^{\alpha_{n}} x_{n}(t)\right)$ is also a solution. This is done in the sequel.

$$
\begin{aligned}
\frac{d}{d t} \bar{x}_{i}(t)=\lambda^{\alpha_{i}} & \frac{d}{d t} x_{i}(t)=\lambda^{\alpha_{i}} F_{i}\left(x_{1}(t), x_{2}(t), \ldots, x_{n}(t)\right) \\
& =F_{i}\left(\lambda^{\alpha_{1}} x_{1}(t), \lambda^{\alpha_{2}} x_{2}(t), \ldots, \lambda^{\alpha_{n}} x_{n}(t)\right)=F_{i}(\bar{x}(t)) .
\end{aligned}
$$

Given the weights $\alpha_{1}, \alpha_{2}, \ldots, \alpha_{n}$ we define the "semi straight line" which passes through a point $x \in \mathbb{R}^{n}$, as

$$
L_{x}=\left\{\left(\lambda^{\alpha_{1}} x_{1}, \lambda^{\alpha_{2}} x_{2}, \ldots, \lambda^{\alpha_{n}} x_{n}\right): \lambda \in \mathbb{R}^{+}\right\} .
$$

Proposition 2.2. Let $F$ be a linear quasi-homogeneous vector field with weights $\alpha_{1}, \alpha_{2}, \ldots, \alpha_{n}$ and consider the differential system $\dot{x}=$ $F(x)$. If $\alpha_{i} \alpha_{j}>0$ for all $i, j=1,2, \ldots, n$, then the knowledge of the solutions near the origin determines the global phase portrait of F. Furthermore, if 0 is locally asymptotically stable, then 0 is a global attractor.

Proof: Clearly we can assume that $\alpha_{i}>0$ for all $i=1,2, \ldots, n$. So the origin is in the closure of $L_{x}$.

Assume that we know the solutions of $\dot{x}=F(x)$ in a neighbourhood $B$ of the origin and let $x$ be a point in $\mathbb{R}^{n}$. Then for $\lambda$ small enough the point $y=\left(\lambda^{\alpha_{1}} x_{1}, \lambda^{\alpha_{2}} x_{2}, \ldots, \lambda^{\alpha_{n}} x_{n}\right)$ belongs to $B$. Let $y(t)$ be the solution of $\dot{x}=F(x)$ which passes through $y$. Then, from Proposition 2.1, we know that

$$
x(t)=\left(\left(\frac{1}{\lambda}\right)^{\alpha_{1}} y_{1}(t),\left(\frac{1}{\lambda}\right)^{\alpha_{2}} y_{2}(t), \ldots,\left(\frac{1}{\lambda}\right)^{\alpha_{n}} y_{n}(t)\right)
$$

is also a solution. And it is clear that this solution passes through $x$. On the other hand it is clear that if the omega limit of $y$ is the origin, the same is true for $x$.

More interesting dynamical behaviours appear for quasi-homogeneous vector fields with weights of different sign (see Sections 3 and 4).

Example. Consider the family of systems

$$
\left\{\begin{array}{l}
\dot{x}=-x \\
\dot{y}=-y+a x^{2} z+b x^{4} \\
\dot{z}=-z+c x^{2}
\end{array}\right.
$$


All these systems are linear quasi-homogeneous with weights $(1,4,2)$. On the other hand, the characteristic polynomial of the linear part is independent of the point and it is equal to $P(\lambda)=(\lambda+1)^{3}$. So we are in the hypothesis of the Markus Yamabe conjecture. Since 0 is locally asymptotically stable from the above result we deduce that 0 is a global attractor.

Let $\dot{x}=F(x)$ and let $x \in \mathbb{R}^{n}$. We say that the solution which passes through $x$ is of exponential type if

$$
x(t)=\left(x_{1} e^{m_{1} t}, x_{2} e^{m_{2} t}, \ldots, x_{n} e^{m_{n} t}\right)
$$

for some $m_{1}, m_{2}, \ldots, m_{n} \in \mathbb{R}$.

Proposition 2.3. Let $F$ be a linear quasi-homogeneous vector field with weights $\alpha_{1}, \alpha_{2}, \ldots, \alpha_{n}$ and let $L_{x}$ be the "semi straight line" which passes through $x$. Then $L_{x}$ is invariant by the flow of $\dot{x}=F(x)$ if and only if the solution which passes through $x$ is of the form $x_{i}(t)=x_{i} e^{m_{i} t}$ where $m_{i}=c \alpha_{i}$ for some $c \in \mathbb{R}$.

Proof: Let $L_{x}=\left\{\left(\lambda^{\alpha_{1}} x_{1}, \lambda^{\alpha_{2}} x_{2}, \ldots, \lambda^{\alpha_{n}} x_{n}\right): \lambda \in \mathbb{R}^{+}\right\}$and consider the parametrization $\lambda=e^{t}$, i.e.,

$$
L_{x}=\left\{\left(e^{\alpha_{1} t} x_{1}, e^{\alpha_{2} t} x_{2}, \ldots, e^{\alpha_{n} t} x_{n}\right): t \in \mathbb{R}^{+}\right\}
$$

If $L_{x}$ is invariant by the flow, then it exists some $\mu(t) \in \mathbb{R}$ such that

$$
\alpha_{i} e^{\alpha_{i} t} x_{i}=\mu(t) F_{i}\left(e^{\alpha_{1} t} x_{1}, e^{\alpha_{2} t} x_{2}, \ldots, e^{\alpha_{n} t} x_{n}\right)
$$

Due to the homogeneity of $F$ this last condition can be written as $\alpha_{i} x_{i}=$ $\mu(t) F_{i}(x)$. Hence $\mu(t) \equiv \mu$ is independent on $t$. Hence

$$
\alpha_{i} x_{i}=\mu F_{i}(x)
$$

If $\mu=0$, then $x=0$. Since 0 is a critical point of $\dot{x}=F(x)$ the solution can also be considered as a solution of exponential type.

If $\mu \neq 0$, consider $x_{i}(t)=x_{i} e^{\frac{\alpha_{i}}{\mu} t}$. Then $x(t)$ is the solution which passes through $x$ :

$$
x_{i}^{\prime}(t)=\frac{\alpha_{i}}{\mu} x_{i} e^{\frac{\alpha_{i}}{\mu} t}=F_{i}(x) e^{\frac{\alpha_{i}}{\mu} t}=F_{i}\left(x_{1} e^{\frac{\alpha_{1}}{\mu} t}, x_{2} e^{\frac{\alpha_{2}}{\mu} t}, \ldots, x_{n} e^{\frac{\alpha_{n}}{\mu} t}\right) .
$$

The last equality is due to the fact that $F$ is a linear quasi-homogeneous vector field with weights $\alpha_{1}, \alpha_{2}, \ldots, \alpha_{n}$. 
Now let $x$ be such that $x_{i}(t)=x_{i} e^{m_{i} t}$ with $m_{i}=c \alpha_{i}$ is a solution of $\dot{x}=F(x)$.

If $c=0$, then $x_{i}(t)=x_{i}$ for all $t \in \mathbb{R}$, and all the points of $L_{x}$ are critical points of $\dot{x}=F(x)$.

If $c \neq 0$, then $x_{i}^{\prime}(t)=F_{i}(x(t))$ implies

$$
c \alpha_{i} x_{i} e^{c \alpha_{i} t}=F_{i}\left(x_{1} e^{c \alpha_{1} t}, x_{2} e^{c \alpha_{2} t}, \ldots, x_{n} e^{c \alpha_{n} t}\right)=e^{c \alpha_{i} t} F_{i}(x) .
$$

Hence $\alpha_{i} x_{i}=(1 / c) F_{i}(x)$. Then, from (4), we see that $L_{x}$ is an invariant straight line.

Remark 2.4. There are some systems which have solutions of exponential type and they are not quasi-homogeneous. For instance, the system

$$
\left\{\begin{array}{l}
\dot{x}=-x+x y-x^{2} y^{2}+2 x^{2} y \\
\dot{y}=y+x^{2}-x^{3} y-2 x y^{2}
\end{array}\right.
$$

has the solution $x(t)=x_{0} e^{t}, y(t)=\left(1 / x_{0}\right) e^{-t}$ and it is not a quasihomogeneous vector field.

If $F$ is a linear quasi-homogeneous vector field with weights $\alpha_{1}, \alpha_{2}, \ldots, \alpha_{n}$ and we look for the solutions of exponential type we have to solve the system of equations

$$
F_{i}(x)=c \alpha_{i} x_{i}, \quad i=1,2, \ldots, n
$$

where $c$ is a real number. This is a system of $n$ quasi-homogeneous equations. It is easy to see that the set of solutions of this type of systems, either, it reduces to the origin or, if $x$ is a solution of the system then each point in $L_{x}$ is also a solution. Solving the above system we can find the invariant "semi straight lines".

Proposition 2.5. Let $F$ be a linear quasi-homogeneous vector field and consider the differential system $\dot{x}=F(x)$. Then the integration of $\dot{x}=F(x)$ in $\mathbb{R}^{n}$ reduces to the integration of a system in $\mathbb{R}^{n-1}$.

Proof: Consider the transformation (outside $x_{1}=0$ )

$$
\begin{aligned}
& y_{1}=x_{1}, \\
& y_{j}=x_{j}^{\alpha_{1}} x_{1}^{-\alpha_{j}}, \quad j \geq 2,
\end{aligned}
$$

and using the homogeneity of $F$ we have that

$$
F_{i}(x)=F_{i}\left(x_{1}, x_{1}^{\frac{\alpha_{2}}{\alpha_{1}}} y_{2}^{\frac{1}{\alpha_{1}}}, \ldots, x_{1}^{\frac{\alpha_{n}}{\alpha_{1}}} y_{n}^{\frac{1}{\alpha_{1}}}\right)=x_{1}^{\frac{\alpha_{i}}{\alpha_{1}}} \tilde{F}\left(y_{2}, y_{3}, \ldots, y_{n}\right)
$$


Then, the system $\dot{x}=F(x)$ reduces to:

$$
\left\{\begin{array}{l}
\dot{y}_{1}=y_{1} \tilde{F}_{1}\left(y_{2}, y_{3}, \ldots, y_{n}\right) \\
\dot{y}_{j}=\alpha_{1} y_{j}^{\frac{\alpha_{1}-1}{\alpha_{1}}} \tilde{F}_{j}\left(y_{2}, y_{3}, \ldots, y_{n}\right)-\alpha_{j} y_{j} \tilde{F}_{1}\left(y_{2}, y_{3}, \ldots, y_{n}\right), \quad j \geq 2 .
\end{array}\right.
$$

Hence, we have that the last $n-1$ equations only depend on the last $n-1$ variables and the first one has separate variables if we already know $y_{j}(t)$ for $j=2,3, \ldots, n$.

Remark 2.6. Let $F$ be a linear quasi-homogeneous vector field and consider the discrete dynamical system generated by $F$. Then, it is easy to obtain similar results to Propositions 2.1, 2.2 and 2.4. In particular the points lying in invariant "semi straight lines" (notice that now invariant means $\left.F\left(L_{x}\right) \subset L_{x}\right)$ have also solutions of exponential type (i.e., $x^{(m)}=$ $\left(x_{1}^{(0)} a_{1}^{m}, x_{2}^{(0)} a_{2}^{m}, \ldots, x_{n}^{(0)} a_{n}^{m}\right)$ for some constants $\left.a_{1}, a_{2}, \ldots, a_{n} \in \mathbb{R}\right)$. In order to find the invariant straight lines we have to solve the system of equations

$$
F_{i}(x)=\lambda^{\alpha_{i}} x_{i}
$$

where $\lambda$ is a real positive number.

\section{Construction of counterexamples}

In this section we will give a classes of polynomial maps which satisfy simultaneously the hypothesis of the MYC and the hypothesis of the DMYQ.

Lemma 3.1. (See $[\mathbf{C G M}]$ ). Let $F$ be a polynomial map from $\mathbb{R}^{n}$ into itself such that for any $x \in \mathbb{R}^{n}, J F(x)$ has all its eigenvalues with modulus less than one. Then the characteristic polynomial of $(D F)_{x}$ is independent on $x$.

Due to Lemma 3.1 we start with simple maps with characteristic polynomial independent on $x$. Particularly we consider maps of type $F(x)=\lambda I+N$, where $\lambda \in \mathbb{R}, I$ is the identity map and $N$ is a nilpotent map (i.e., $(D N)_{x}$ has all its eigenvalues equal zero at each $\left.x \in \mathbb{R}^{n}\right)$. Then, $(D F)_{x}$ has all its eigenvalues equal $\lambda$ at each $x \in \mathbb{R}^{n}$. Hence, we will consider $\lambda<0$ (resp. $|\lambda|<1$ ) for the continous (resp. discrete) problem.

Assume that $n=2$. Then $N$ nilpotent implies that

$$
\frac{\partial N_{1}}{\partial x}+\frac{\partial N_{2}}{\partial y} \equiv 0 \quad \text { and } \quad \frac{\partial N_{1}}{\partial x} \frac{\partial N_{2}}{\partial y}-\frac{\partial N_{1}}{\partial y} \frac{\partial N_{2}}{\partial x} \equiv 0 .
$$


So, it exists some $H(x, y)$ with $N_{1}(x, y)=\frac{-\partial H}{\partial y}(x, y), N_{2}=\frac{\partial H}{\partial x}(x, y)$ and the hessian of $H$ is identically zero. From a classical result of differential geometry we have that these type of maps can be written, through an affine transformation, as $H(u, v)=u+g(v)$ (see $[\mathbf{C}]$ and also [D]). Hence, $H(x, y)=\alpha x+\beta y+\gamma+g(a x+b y+c)$ provide us a family of MY examples in $\mathbb{R}^{2}$ :

$$
F(x, y)=(\lambda x-b f(a x+b y+c), \lambda y+a f(a x+b y+c))
$$

where $f=g^{\prime}$, which can be extended to $\mathbb{R}^{n}$ for $n \geq 3$ by

$$
F(x)=\left(\lambda x_{1}-b\left(x_{3}\right) f(u), \lambda x_{2}+a\left(x_{3}\right) f(u), \lambda x_{3}, \ldots, \lambda x_{n}\right)
$$

with $u=a\left(x_{3}\right) x_{1}+b\left(x_{3}\right) x_{2}+c\left(x_{3}\right)$ and $a, b, c$ arbitrary smooth functions of $x_{3}$.

Taking $a\left(x_{3}\right)=a x_{3}^{l}, b\left(x_{3}\right)=b x_{3}^{m}$ and $f(u)=u^{k}(a, b \in \mathbb{R}, k, l, m \in \mathbb{N})$ we obtain the following:

Theorem 3.2. The family of maps

$$
\begin{aligned}
& F(x)=\left(\lambda x_{1}-b x_{3}^{m}\left(a x_{1} x_{3}^{l}+b x_{2} x_{3}^{m}\right)^{k},\right. \\
& \left.\quad \lambda x_{2}+a x_{3}^{l}\left(a x_{1} x_{3}^{l}+b x_{2} x_{3}^{m}\right)^{k}, \lambda x_{3}, \ldots, \lambda x_{n}\right)
\end{aligned}
$$

satisfy the following properties:

(1) For all $a, b, \lambda \in \mathbb{R}, k, l, m \in \mathbb{N}$ they are linear quasi-homogeneous with weights

$$
\left(\alpha_{1}, \alpha_{2}, \ldots, \alpha_{n}\right)=(m+k l, l+k m, 1-k, \ldots, 1-k) .
$$

(2) For all $\lambda \in \mathbb{R}$ with $\lambda<0$ (resp. $|\lambda|<1$ ) they satisfy the hypothesis of the $M Y C$ (resp. the DMYQ).

(3) For all $\lambda \in \mathbb{R}$ with $\lambda<0$ (resp. $|\lambda|<1$ ) $k$ an even number, $l, k, l-$ $m \in \mathbb{N}$ different from zero and for all $a, b \in \mathbb{R}$ the differential system $\dot{x}=F(x)$ (resp., the discret dynamical system generated by $F$ ) has unbounded orbits.

Proof: The proof of (1) and (2) is straightforward. In order to see (3) we begin considering the dynamical system $\dot{x}=F(x)$. The system of equations $F_{i}(x)=c \alpha_{i} x_{i}$ writes as:

$$
\left\{\begin{array}{l}
\lambda x_{1}-b x_{3}^{m}\left(a x_{1} x_{3}^{l}+b x_{2} x_{3}^{m}\right)^{k}=c(m+k l) x_{1} \\
\lambda x_{2}+a x_{3}^{l}\left(a x_{1} x_{3}^{l}+b x_{2} x_{3}^{m}\right)^{k}=c(l+k m) x_{2} \\
\lambda x_{i}=c(1-k) x_{i}, \quad i=3,4, \ldots, n
\end{array}\right.
$$


which gives the solutions

(6)

$$
\left\{\begin{array}{l}
c=\frac{\lambda}{1-k}>0 \\
x_{1}=\frac{-b B}{a A} x_{2} x_{3}^{m-l} \\
x_{2}^{k-1} x_{3}^{l+k m}=\frac{-B}{a}\left(\frac{A}{b \lambda(l-m)}\right)^{k}
\end{array}\right.
$$

where $A=\lambda \frac{(1-k)-(m+k l)}{1-k}$ and $B=\lambda \frac{(1-k)-(l+k m)}{1-k}$. It is clear that system (5) has always real solutions. Let $\bar{x}_{1}, \bar{x}_{2}$ and $\bar{x}_{3}$ be one of them and let $\bar{x}_{i}$ be arbitrary real numbers for $i=4,5, \ldots, n$. Then

$$
\left\{\begin{array}{l}
x_{1}(t)=\bar{x}_{1} e^{\frac{\lambda(m+k l)}{1-k} t} \\
x_{2}(t)=\bar{x}_{2} e^{\frac{\lambda(l+k m)}{1-k} t} \\
x_{i}(t)=\bar{x}_{i} e^{\lambda t}, \quad i=3,4, \ldots, n
\end{array}\right.
$$

is a solution of $\dot{x}=F(x)$. And it is clear that $x_{1}(t), x_{2}(t) \rightarrow \infty$ as $t \rightarrow \infty$.

Now consider the discret dynamical system generated by $F$. From Remark 2.6 we have to solve the system of equations $F_{i}(x)=\mu^{\alpha_{i}} x_{i}$, i.e.,

$$
\left\{\begin{array}{l}
\lambda x_{1}-b x_{3}^{m}\left(a x_{1} x_{3}^{l}+b x_{2} x_{3}^{m}\right)^{k}=\mu^{m+k l} x_{1} \\
\lambda x_{2}+a x_{3}^{l}\left(a x_{1} x_{3}^{l}+b x_{2} x_{3}^{m}\right)^{k}=\mu^{l+k m} x_{2} \\
\lambda x_{i}=\mu^{1-k} x_{i}, \quad i=3,4, \ldots, n
\end{array}\right.
$$

which gives the solutions

$$
\left\{\begin{array}{l}
\mu=\lambda^{\frac{1}{1-k}} \\
x_{1}=\frac{-b D}{a C} x_{2} x_{3}^{m-l} \\
x_{2}^{k-1} x_{3}^{l+k m}=\frac{-D}{a}\left(\frac{C}{b(C-D)}\right)^{k}
\end{array}\right.
$$

where $C=\lambda-\lambda^{\frac{m+k l}{1-k}}$ and $D=\lambda-\lambda^{\frac{l+k m}{1-k}}$. Notice that $|\mu|>1$. As before take $\bar{x}_{1}, \bar{x}_{2}$ and $\bar{x}_{3}$ a solution of (7) and $\bar{x}_{i}$ arbitrary real numbers for $i=4,5, \ldots, n$. Then

$$
\left\{\begin{array}{l}
x_{1}^{(n)}=\bar{x}_{1}\left(\lambda^{\frac{m+k l}{1-k}}\right)^{n} \\
x_{2}^{(n)}=\bar{x}_{2}\left(\lambda^{\frac{l+k m}{1-k}}\right)^{n} \\
x_{i}^{(n)}=\bar{x}_{i}(\lambda)^{n}, \quad i=3,4, \ldots, n
\end{array}\right.
$$

gives the complete orbit which begins in $\bar{x}=\left(\bar{x}_{1}, \bar{x}_{2}, \ldots, \bar{x}_{n}\right)$ and clearly $x_{1}^{(n)}, x_{2}^{(n)} \rightarrow \infty$ as $n \rightarrow \infty$. 


\section{On the global dynamics of simple counterexamples}

One of the easiest examples of map $F(x)$ given in Proposition 3.2 is

(8) $F\left(x_{1}, x_{2}, x_{3}\right)=\left(-x_{1}+x_{3}\left(x_{1}+x_{2} x_{3}\right)^{2},-x_{2}-\left(x_{1}+x_{2} x_{3}\right)^{2},-x_{3}\right)$.

The above map is the one considered in [CEGHM]. The goal of this section is firstly to describe the global phase portrait of the equation $\dot{x}=F(x)$ and secondly to study an analogous discrete problem. lem.

The following theorem is the main result concerning to the first prob-

Theorem 4.1. Consider the initial value problem

$$
\left\{\begin{array}{l}
\dot{x}=F(x) \\
x(0)=x^{0}=\left(x_{1}^{0}, x_{2}^{0}, x_{3}^{0}\right)
\end{array}\right.
$$

where $F(x)$ is given in (8), and denote by $\phi\left(t, x^{0}\right)$ its solution defined in the maximal interval $I_{x^{0}}=\left(\alpha\left(x^{0}\right), \beta\left(x^{0}\right)\right)$. Then

(1) The vector field defined by F satisfies the hypothesis of the Markus- Yamabe Conjecture.

(2) Define $v^{0}=\left(x_{1}^{0}+x_{2}^{0} x_{3}^{0}\right) x_{3}^{0}, w^{0}=x_{2}^{0}\left(x_{3}^{0}\right)^{2}$. Then

$$
\lim _{t \rightarrow \beta\left(x^{0}\right)}\left\|\phi\left(t, x^{0}\right)\right\|= \begin{cases}0, & \text { if }\left(v^{0}, w^{0}\right) \in W^{s}(0,0) \\ \infty, & \text { if }\left(v^{0}, w^{0}\right) \notin W^{s}(0,0)\end{cases}
$$

where $W^{s}(0,0)$ denotes the basin of attraction of the origin for the system

$$
(\dot{v}, \dot{w})=\left(-2 v-w,-3 w-v^{2}\right) .
$$

Proof: The proof of (1) is a direct consequence of Proposition 3.2 taking $a=b=-1$.

If we search for invariant "semi straight lines" of system (8) we see that $L_{x}$ is invariant if and only if $\left(x_{1}, x_{2}, x_{3}\right) \in L_{( \pm 18,-12, \pm 1)}$. In fact, $x_{1}(t)= \pm 18 e^{t}, x_{2}(t)=-12 e^{2 t}, x_{3}(t)= \pm e^{-t}$ are two solutions of (8) which clearly are not bounded (we notice that the solution given in $[$ CEGHM] is one of them).

On the other hand we use Proposition 2.5 to reduce the dimension of the system outside the invariant plane $x_{3}=0$. To be exact consider the 
quasi-homogeneous change of variables $H\left(x_{1}, x_{2}, x_{3}\right)=(v, w, z)$, outside $x_{3}=0$, defined by

$$
(v, w, z)=\left(\left(x_{1}+x_{2} x_{3}\right) x_{3}, x_{2} x_{3}^{2}, x_{3}\right) .
$$

Thus (8) is transformed into

$$
(\dot{v}, \dot{w}, \dot{z})=\left(-2 v-w,-3 w-v^{2},-z\right)
$$

Now consider the planar quadratic system

$$
(\dot{v}, \dot{w})=\left(-2 v-w,-3 w-v^{2}\right) .
$$

The global dynamics of $(12)$ in the $(v, w)$-plane can be studied without major difficulties. We describe it in the sequel (see also Figure 1): There are two critical points, an attracting node at $O=(0,0)$ and a hyperbolic saddle at $S=(6,-12)$. The unstable separatrices of the saddle point are contained in the invariant algebraic curve $2 v^{3}-3 w^{2}=0$. The stable separatrices of this point have empty $\alpha$-limit (they come from a critical point at infinity) and are the boundary between the basin of attraction of the attracting node, $W^{s}(0,0)$, and the set of points which have $\alpha$-limit and $\omega$-limit empty (except for the unstable separatrix of the saddle). Moreover for these points we have that $w(t)$ tends to infinity when $t$ tends to right endpoint of the interval of definition of the solution.

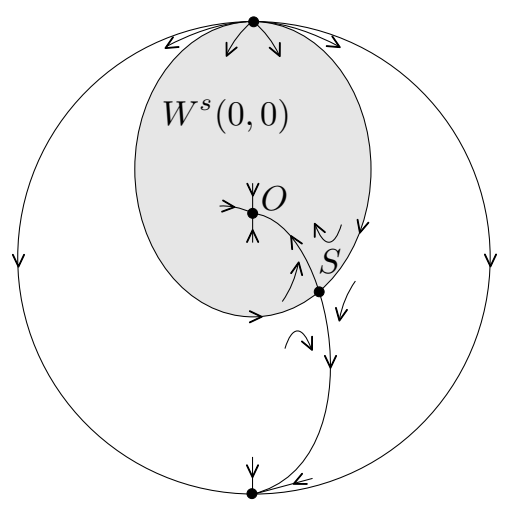

Figure 1. Phase portrait of (12) in the Poincaré disk.

Take $x^{0} \in \mathbb{R}^{3}$ such that $\left(v^{0}, w^{0}\right) \in W^{s}(0,0)$. We want to prove that $\lim _{t \rightarrow \omega\left(x^{0}\right)} \phi\left(t, x^{0}\right)=0$. If $x_{3}^{0}=0$ the proof is trivial. If $x_{3}^{0} \neq 0$, unmaking 
the change of variables (11) we have that

$$
\begin{aligned}
& u(t)=\frac{v(t)}{x_{3}^{0} e^{-t}}, \\
& y(t)=\frac{w(t)}{\left(x_{3}^{0} e^{-t}\right)^{2}} .
\end{aligned}
$$

Since $v(t)$ and $w(t)$ are defined for all $t \geq 0$ we get that $\omega\left(x^{0}\right)=\infty$ and hence we need to prove that $\lim _{t \rightarrow \infty} \phi\left(t, x^{0}\right)=0$. In other words we have to compute

$$
\lim _{t \rightarrow \infty} \frac{v(t)}{x_{3}^{0} e^{-t}}, \quad \text { and } \quad \lim _{t \rightarrow \infty} \frac{w(t)}{\left(x_{3}^{0} e^{-t}\right)^{2}} .
$$

Since for system (12) the eigenvalues of the node are -2 and -3 we can apply Poincaré's Theorem (see [AA, p. 72] which ensures that (12) is in a neighbourhood of the origin, analytically conjugate to its linear part at the origin. That is the speed of convergence at zero of $v(t)$ and $w(t)$ is at least $e^{-2 t}$. Therefore

$$
\lim _{t \rightarrow \infty}(u(t), y(t), z(t))=(0, k, 0)
$$

for some real number $k$. Considering the first change of variables we get

$$
\lim _{t \rightarrow \infty} x(t)=(0, k, 0)
$$

Since $(0,0,0)$ is the only critical point of $(8)$, we obtain that $k=0$ and we have concluded this part of the proof.

Now let $x^{0} \in \mathbb{R}^{3}$ be such that $\left(v^{0}, w^{0}\right) \notin W^{s}(0,0)$. Clearly in this case $x_{3}^{0} \neq 0$. If $\omega\left(x^{0}\right) \neq \infty$ then $\lim _{t \rightarrow \omega\left(x^{0}\right)}\|x(t)\|=\infty$ so we assume that $\omega\left(x^{0}\right)=\infty$. Then we get

$$
\lim _{t \rightarrow \infty}|| x(t) \| \geq \lim _{t \rightarrow \infty}\left|x_{2}(t)\right|=\lim _{t \rightarrow \infty} \frac{w(t)}{\left(x_{3}^{0} e^{-t}\right)^{2}}=\infty
$$

Remark 4.2. Let us call $V^{s}(0)$ the basin of attraction of 0 for system (8). In order to describe it we notice that the transformation $H\left(x_{1}, x_{2}, x_{3}\right)=(v, w, z)$ given in (10) is a diffeomorphism between the half space $x_{3}>0$ (resp. $\left.x_{3}<0\right)$ and $z>0$ (resp. $\left.z<0\right)$. Furthermore it satisfies

$$
H\left(L_{\left(x_{1}^{0}, x_{2}^{0} \cdot x_{3}^{0}\right)}\right)=\left\{\left(v_{0}, w_{0}, z\right), z x_{3}^{0}>0\right\} .
$$


So each vertical line $\left(v_{0}\right.$ and $w_{0}$ fixed $)$ in the space $(v, w, z)$ splits into two "semi straight lines" in the space $\left(x_{1}, x_{2}, x_{3}\right)$. This fact and the result of Theorem 4.1 let us to see that $\partial V^{s}(0)=H^{-1}(\{(v, w, z),(v, w) \in$ $\left.\partial W^{s}(0,0), z \neq 0\right\}$ (where $\partial E$ denotes the topological boundary of $E$ ) and hence, this boundary has two connected components.

Remark 4.3. The solution of (8) found in [CEGHM] for proving that the origin is not a global attractor is the one corresponding to $\left(v^{0}, w^{0}\right)=(6,-12)$ that is, the saddle point of $(12)$, which is in the boundary of $W^{s}(0,0)$.

Remark 4.4. It is easy to test that $\left(2 v^{3}-3 w^{2}\right)^{-5 / 6}$ is an integrating factor for the vector field defined by (12). Therefore a first integral can be found for (12). The effective computation of this first integral needs to know a primitive of the function $(a x+b)^{-5 / 6} x^{-2 / 3}$. It is well known (see for instance $[\mathbf{C h}]$ ) that this primitive cannot be expressed in terms of elementary functions. Therefore it seems natural to believe that the boundary of $W^{s}(0,0)$ cannot be expressed in terms of elementary functions.

Remark 4.5. Another way to describe the global dynamics of (9) was suggested us by our colleague Xavier Chavarriga. It consists first in considering the transformation $(v, w, z)=(u / z, y / z, z)$ and later $(h, w, z)=$ $\left(v^{3} / 3-w^{2} / 2, w, z\right)$ applied to (10). With these last variables (10) writes as $(\dot{h}, \dot{w}, \dot{z})=\left(0,-\left(3 h+3 / 2 w^{2}\right)^{2 / 3} z,-z\right)$ and it can be easily studied. In particular the solution found in [CEGHM] lives inside the invariant surface $h=0$, where the flow of (9) can be explicitly obtained from the above expression. We have choosed our approach because it can be applied to any quasi-homogeneous vector field (see Proposition 2.5) while the above change is thought just for simplifying system (9).

A similar result to Theorem 4.1 can be presented to describe the global dynamics of the counterexample to the discrete Markus-Yamabe question given in $[$ CEGHM] by the map

$$
F\left(x_{1}, x_{2}, x_{3}\right)=\left(\frac{1}{2} x_{1}+x_{3}\left(x_{1}+x_{2} x_{3}\right)^{2}, \frac{1}{2} x_{2}-\left(x_{1}+x_{2} x_{3}\right)^{2}, \frac{1}{2} x_{3}\right) .
$$

Theorem 4.6. Consider the discrete dynamical system generated by $F$ given in (13). That is

$$
\left\{\begin{array}{l}
x^{n+1}=F\left(x^{n}\right) \\
x^{0}=\left(x_{1}^{0}, x_{2}^{0}, x_{3}^{0}\right) \in \mathbb{R}^{3}
\end{array}\right.
$$


Then

(1) The map $F$ is under the hypothesis of the discrete Markus-Yamabe Question.

(2) Define $v^{0}=\left(x_{1}^{0}+x_{2}^{0} x_{3}^{0}\right) x_{3}^{0}$ and $w^{0}=x_{2}^{0}\left(x_{3}^{0}\right)^{2}$. Then

$$
\lim _{n \rightarrow \infty}\left\|x^{n}\right\|= \begin{cases}0 & \text { if }\left(v^{0}, w^{0}\right) \in W^{s}(0,0) \\ \infty & \text { if }\left(v^{0}, w^{0}\right) \notin W^{s}(0,0)\end{cases}
$$

where $W^{s}(0,0)$ denotes the basin of attraction of the origin for the discrete dynamical system generated by

$$
G(v, w)=\left(v / 4-w / 8+v^{2} / 4, w / 8-v^{2} / 4\right)
$$

The proof of this last theorem follows the same steps that the proof of the Theorem 4.1. The major difference consists in checking that the two eigenvalues of the attracting node of the discrete dynamical system generated by $(14),(0,0)$ are $1 / 4$ and $1 / 8$ and so the critical point is under the hypothesis of Poincaré's Theorem for diffeomorphisms (see [AA, p. 99]).

Remark 4.7. The basin of attraction of the origin for system (12) is well described in the proof of Theorem 4.1 and in Figure 1. On the other hand the description of this basin for the discrete dynamical system generated by (14) is not so easy. We do not study this problem here.

\section{References}

[AA] D. V. Anosov And V. I. Arnold, "Dynamical Systems I," Enciclopaedia of Mathematical Sciences, Springer-Verlag, 1985.

[A] V. I. ARnOLD, "Chapitres supplémentaires de la théorie des équations différentielles ordinaries," Ed. MIR, Moscow, 1980.

[B] N. Barabanov, On a problem of Kalman, Siberian Mathematical Journal 29 (1988), 333-341.

[BDST] H. W. Broer, F. Dumortier, S. J. VAn Strien AND F. TAkens, "Structures in Dynamics," Studies in Math. Physics 2, (E. M. de Jager, Ed.), North-Holland, 1991. 
[BL] J. Bernat and J. Llibre, Counterexamples to Markus Yamabe Conjecture, Dynamics of Continuous, Discrete and Inpulsive Systems 2 (1996), 337-379.

[C] M. P. Do Carmo, "Differential Geometry of Curves and Surfaces," Prentice Hall, 1976.

[Ch] P. L. Chebychev, Sur l'integration des differentielles irrationelles, J. Math. Pures Appl. 18 (1853), 87-11.

[CEGHM] A. Cima, A. van den Essen, A. Gasull, E. Hubbers AND F. MAÑosas, A counterexample to the Markus Yamabe Conjecture, Report of University of Nijmegen 9551 (1995), to appear in Adv. Math..

[CGM] A. Cima, A. Gasull and F. Mañosas, The discret Markus Yamabe problem, Prepublicacions de l'UAB 26 (1995).

[D] F. Dillen, Polynomials with constant hessian determinant, J. of Pure and App. Algebra 71 (1991), 13-18.

[EH] A. VAn DEN Essen AND E. HubBers, Chaotic polynomial automorphisms; counterexamples to several conjectures, Report of University of Nijmegen 9549 .

[F] R. Fessler, A Proof of the two-dimensional Markus-Yamabe Stability Conjecture, Annales Polonici Mathematici 62 (1995), 45-75.

[G1] A. A. GLutsuk, The complete solution of the Jacobian problem for vector fields on the plane, Comm. Moscow Math. Soc., Russian Math. Surveys 49 (1994), 185-186.

[G2] A. A. Glutsuk, Asymptotic stability of linearizations of a vector field in $\mathbb{R}^{3}$ with a singular point does not imply global stabililty, Preprint (1996).

[Gu] C. Gutierrez, A solution to the bidimensional Global Asymptotic Conjecture, Ann. Inst. H. Poincaré Anal Non Linéaire 12 (1995), 627-671.

[MO] G. Meisters And C. Olech, Solution of the global asymptotic stability jacobian conjecture for the polynomial case, Analyse Mathématique et Applications (1988), 1-9. 
[MY] L. Markus And H. Yamabe, Global stability criteria for differential systems, Osaka Math. Journal 12 (2) (1960), 305-317.

[S] J. P. La SAlle, The stability of Dynamical Systems, CBMS-NSF Regional Conference Series in Applied Math. 25, SIAM 1976, 81 pages, 2nd printing 1993.

\author{
Anna Cima: \\ Departament de Matemàtica Aplicada II \\ E. T. S. d'Enginyers Industrials \\ Universitat Politècnica de Catalunya \\ Colom 11 \\ 08222 Terrassa (Barcelona) \\ SPAIN \\ e-mail: cima@ma2.upc.es
}

\author{
Armengol Gasull: \\ Departament de Matemàtiques \\ Universitat Autònoma de Barcelona \\ 08193 Bellaterra (Barcelona) \\ SPAIN \\ e-mail: gasull@mat.uab.es
}

Francesc Mañosas:

Departament de Matemàtiques

Universitat Autònoma de Barcelona

08193 Bellaterra (Barcelona)

SPAIN

e-mail: manyosas@mat.uab.es

Rebut el 30 de Novembre de 1996 